\title{
KILOMETER-SQUARE AREA RADIO SYNTHESIS TELESCOPE
}

$K A R S T$ project

\author{
B. PENG AND R. NAN
}

Beijing Astronomical Observatory, China

\section{Introduction}

One way to realize the Large radio Telescope with a collecting area approaching one square kilometer, continuously covering a frequency range between $200 \mathrm{MHz}$ and $2 \mathrm{GHz}$, is to construct a passive spherical reflector array of about 30 individual unit telescopes, each $\sim 300 \mathrm{~m}$ diameter(Butcher 1995). Valleys amid the hills of southwest China would be ideal for such LT concept. We refer to this effort as the Kilometer-square Area Radio Synthesis Telescope project. Site surveying and Radio Interference monitoring looks promising.Engineering considerations are summarized.

\section{The Site, RIF Monitoring and Engineering Considerations}

A large number of karst depressions, more than 400 , have been found in the south Guizhou. There are less than 5 days of snowfall, and no ice build-up at the sites. Statistical results (Figure 1) by using remote sensing, geographic information system techniques and on-the-spot observation provide suitable sites.

A series of measurements at various sites in Guizhou have been carried out to check on their suitability, from the point of view of interference for realizing the KARST. The first measurements were made at 8 karst depression sites in Nov. 1994 in Pingtang and Puding counties. In addition we monitored a site in the center of Guiyang and at the Urumqi astronomical station for comparison. Additional measurements were made in March 1995 in an attempt to understand distance effects. The RIF monitoring results (Peng et al. 1996) look quite promising. Remoteness together with the futility of industrial development potentially benefit the RIF environment.

A backup structure for the main spherical reflector could be composed of ring-radial beams of concrete (Wang 1996). A synthetic design with 

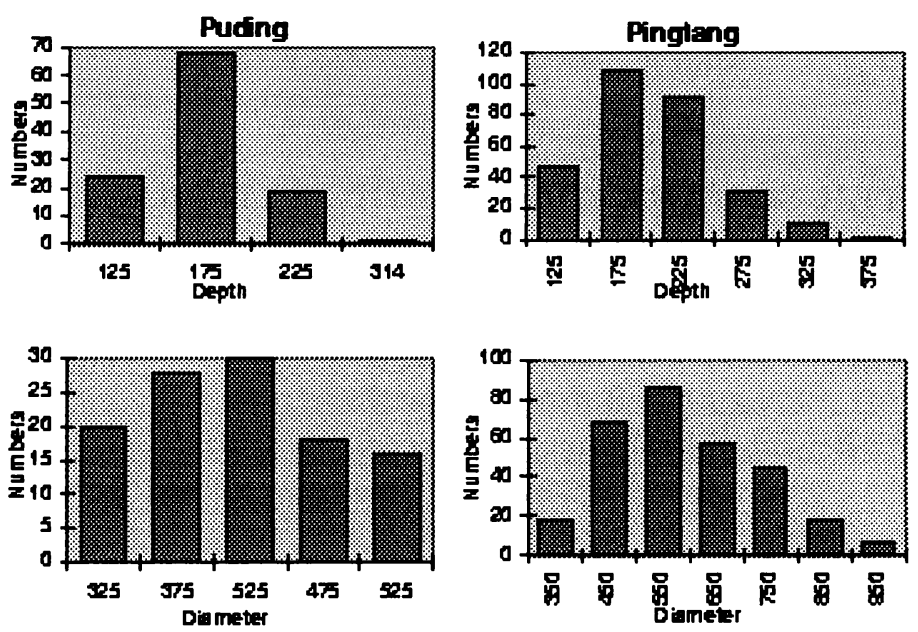

Figure 1. Statistical results at Pingtang and Puding counties

mechanics and electronics is proposed (Duan 1996), in which the line feed is supported by six suspended cables connected to a mechanical servo system controlled by a computer, and the ends of the line feed move along the two spherical surfaces. Laser techniques would be employed to accurately detect the position of the line feed in time. Line feeds (Mao \& Jiao 1996), dual reflectors with a phased array (Guo 1996), and hybrid feeds (Xiong \& Xie 1996) consisting of a co-spheric array and a line feed which diminishes the length of a single line feed required and reduces the blockage effect were it a single array feed have been discussed at an elementary level for the spherical reflectors. Several problems such as mutual coupling effects, overlapping and spillover are expected to be studied in more detail.

\section{References}

Butcher H., 1995. Astron NFRA. Issue no.9 page 5-7.

Duan B., Zhao Y., Wang J. and Xu G., 1996. Proc. of the 3rd Meeting of the Large Telescope Working Group and of a Workshop on Spherical Radio Telescope, eds: Richard Strom, Bo Peng and Rendong Nan 85-102.

Guo Y., Shu X. and Yan, H., 1996. Proc. of the LTWG-3 \& W-SRT. eds: Richard Strom, Bo Peng and Rendong Nan 175-177

Mao Y. and Y. Jiao Y., 1996. Proc. of the LTWG-3 \& W-SRT. 81-84

Peng B., Strom R., Nan R., Nie Y., Piao T., Kang L., Yan Y. and Wu S., 1996. Proc. of the LTWG-3 \& W-SRT. 144-151

Wang J., Xu G., Duan B., and Li H., 1996. Proc. of the LTWG-3 \& W-SRT. 158-159

Xiong J. \& Xie S., 1996. Proc. of the LTWG-3 \& W-SRT. 116-122 